\title{
Clinical Factors Associated with Dense and Wedge-Shaped Nephrograms Detected 24 h After Chemoembolization
}

\author{
Wayne L. Monsky $\cdot$ Anokh Pahwa • \\ Chin-Shang Li · Richard W. Katzberg
}

Received: 17 February 2009/Accepted: 28 July 2009/Published online: 17 September 2009

(c) The Author(s) 2009. This article is published with open access at Springerlink.com

\begin{abstract}
This investigation aimed to evaluate patient characteristics and procedural factors associated with abnormal nephrograms encountered on noncontrast computed axial tomography (CAT) obtained 24-h after transarterial chemoembolization (TACE) for primary and metastatic hepatic malignancies. Sixty hepatic chemoembolization procedures were performed in 29 patients who had a median age of 63 years (range 42-79). The male-tofemale ratio was 16:13. Noncontrast CAT scans were obtained approximately $24 \mathrm{~h}$ after TACE as part of our institutional protocol and were examined for persistent renal nephrograms. These findings were compared with clinical and procedural parameters to determine whether there was any association with these factors or with the occurrence of acute renal failure (ARF). Abnormally persistent CAT nephrograms were observed $24 \mathrm{~h}$ after 28 of 60 (46.7\%) TACE procedures, of which 14 (23.3\%) were persistent, bilaterally dense, global nephrograms, and 14 (23.3\%) were small, wedge-shaped, and focal nephrograms. The change in serum creatinine from baseline to $24 \mathrm{~h}$ was significantly greater $(p=0.031)$ in the global nephrogram group. The presence of cirrhosis, Child-Pugh score, procedure time, baseline renal insufficiency, and lower periprocedural mean arterial blood pressure were also statistically significantly associated with the occurrence of bilateral globally dense nephrograms. The procedure time was
\end{abstract}

W. L. Monsky · A. Pahwa · R. W. Katzberg $(\bowtie)$

Department of Radiology, University of California Davis

Medical Center, Sacramento, CA 95817, USA

e-mail: richard.katzberg@ucdmc.ucdavis.edu

C.-S. Li

Division of Biostatistics, Department of Public Health Sciences,

University of California, Davis, CA, USA statistically significantly associated with the occurrence of wedge-like focally persistent nephrograms. Global, persistently dense nephrograms and wedge-shaped focally persistent nephrograms are not infrequently observed after TACE. Persistent global nephrograms can be an important clinical indicator of ARF. The wedge nephrogram may represent focal renal ischemia.

Keywords Interventional radiology $\cdot$ Kidney · Kidney nephrograms · MDCT · Chemoembolization · Kidney injury

\section{Introduction}

As part of our institutional protocol, a nonenhanced abdominal computed axial tomography (CAT) scan is obtained $24 \mathrm{~h}$ after transarterial chemoembolization (TACE) for primary and metastatic hepatic malignancies. The rationale for these CAT scans is to evaluate the technical success of the procedure: primarily to document distribution of the chemoembolization agents suspended in ethiodol and secondarily to exclude the possibility of nontarget embolization of these agents in the mucosa of the intestinal tract or gallbladder. On the 24-h noncontrast CAT scans, we occasionally observe two types of unusual nephrographic patterns: diffuse, bilateral, and globally dense nephograms or wedge-shaped and focally dense nephograms. The few previous descriptions of these nephrograms in the literature suggest that the global nephrogram can be an indicator of acute renal failure (ARF) and the wedge-like, segmental nephrogram may indicate focal renal ischemia [1-6].

The purpose of this retrospective investigation was to determine the frequency with which the global and wedge- 
shaped nephrograms seen after TACE procedures are detected on follow-up noncontrast CAT and to assess whether they are associated with changes in renal function. Furthermore, we aimed to investigate any clinical factors associated with these findings.

\section{Materials and Methods}

Institutional Review Board approval was granted for the retrospective review of the electronic medical records and archived CAT and angiographic images. From 2005 to 2007, 64 consecutive TACE procedures of the liver were performed in 30 patients. One patient and 4 CAT scans were excluded because emergent contrast CAT scans were required to be performed within $12 \mathrm{~h}$ of the TACE procedures. Our clinical material describes the findings derived from 29 patients undergoing 60 noncontrast CAT scans $24 \mathrm{~h}$ after TACE. Characteristics of the patient population are shown in Table 1. Twenty-one hepatocellular carcinomas, 4 carcinoids, 1 insulinoma, and 3 colorectal carcinomas were treated, with the tumor burden being defined as the sum measurement of the tumor's longest diameters according to the Response Evaluation Criteria in Solid Tumors (RECIST) guidelines [7].

\section{Technical Procedure}

Transfemoral access using a $5 \mathrm{~F}$ vascular sheath was obtained for all patients, and a $5 \mathrm{~F}$ angiographic catheter (Cobra C2 glide catheter [Terumo, Somerset, NJ] or Simmons 1 Catheter [Cook, Bloomington IN]) was used for angiography. Contrast media (CM) used were either iohexol-350 (Omnipaque; $350 \mathrm{mgI} / \mathrm{mL}$ ) or iodixanol-320 (Visipaque; $320 \mathrm{mgI} / \mathrm{mL}$; GE Healthcare, Princeton, NJ). The first step was to document the arterial supply to the liver. The mesenteric arteries were examined to visualize any variant blood supply to the liver. The common hepatic

Table 1 Summary of patient demographics

\begin{tabular}{ll}
\hline Characteristic & $n$ \\
\hline Patients & 29 \\
Procedures & 60 \\
Average age \pm SD (y) & $62 \pm 10$ \\
Median/range age (y) & $63 / 42-79$ \\
M/F & $16 / 13$ \\
Indication for TACE & \\
HCC & 21 \\
CRC & 3 \\
Insulinoma & 1 \\
Carcinoid & 4 \\
\hline
\end{tabular}

artery was then selected, and the catheter was placed in a position distal to the origin of the gastroduodenal artery in the right or left lobar artery. If a stable catheter position was not achieved at this level, or if it was believed that the catheter was blocking forward arterial flow, or if more selective intratumoral delivery was desired, a $2.7-\mathrm{F} \mathrm{mi-}$ crocatheter (Renegade Hi-Flow, Boston Scientific [Natick, MA] or Prograte [Terumo, Somerset, NJ]) and wire (.018inch Agility GT [Terumo] or a .016-inch Fathom [Boston Scientific]) were used for super-selective catheterization of the segmental vessels supplying the tumors.

After informed patient consent was obtained, TACE was performed according to our institutional protocol as described by Solomon et al. [8]. Briefly, doxorubicin (60 mg), mitomycin-C (10 mg), and cisplatin $(100 \mathrm{mg})$ were suspended in ethiodized oil (Ethiodol; Savage Laboratories, Melville, NY) for delivery. This suspension was agitated into a slurry. Approximately one vial of $\sim 200$ 300 micron embolic agent was added (Embosphere, Biosphere Medical, Rockland, Maryland), Contor SE PVA, (Boston Scientific, Natick Massachusetts) or LC Bead (Angiodynamics, Freemont, CA). The treatment was terminated after the total dose was administered and/or near stasis was attained in the appropriate arterial anatomy without reflux. Periprocedural nausea, pain, or fever that occurred in association with TACE was managed symptomatically with antiemetics, e.g., intravenous Phenergan $25 \mathrm{mg}$ (Baxter, Deerfield, IL), analgesics (morphine or patient-controlled Dilaudid [Abbott Laboratories, Abbott Park, IL]), and/or antipyretics. Periprocedural antibiotics (500 mg Rocephin [Hoffmann-La Roche Inc., Nutley, NJ] and $1 \mathrm{~g}$ Flagyl [Pfizer Inc., NY]) were given. Vigorous periprocedural hydration (e.g., $200 \mathrm{cc} / \mathrm{h}$ normal saline) was initiated unless contraindicated, e.g., history of congestive heart failure. Usually a total of one liter of fluids is given before and during the procedure with an additional liter given after the procedure. If the baseline serum creatinine (SCr) was $\geq 1.5 \mathrm{mg} / \mathrm{dL}$, patients received $154 \mathrm{mEq} / \mathrm{L}$ sodium bicarbonate in dextrose and $\mathrm{H}_{2} \mathrm{O}$ - prepared in the hospital pharmacy by adding $154 \mathrm{~mL} 1000 \mathrm{mEq} / \mathrm{L}$ sodium bicarbonate to $846 \mathrm{~mL} \mathrm{5 \%}$ dextrose in $\mathrm{H}_{2} \mathrm{O}$ - as a bolus of $3 \mathrm{~mL} / \mathrm{kg} / \mathrm{h}$ for $1 \mathrm{~h}$ before the procedure, followed by an infusion of $1 \mathrm{~mL} / \mathrm{kg} / \mathrm{h}$ for $6 \mathrm{~h}$ after the procedure, in accordance with Merten et al. [9].

If any patient experienced signs or symptoms of postTACE syndrome, as manifested by nausea and vomiting, continued abdominal pain, and fever, he or she was admitted overnight for continued observation. If symptoms persisted, the patients remained hospitalized until symptoms improved and they were believed to be clinically stable, thus warranting discharge. Each patient was closely monitored, which entailed additional laboratory testing, including a 48-h serum creatinine determination. 
Noncontrast 24-h CAT Image Acquisition and Renal Attenuation Analysis

Approximately $24 \mathrm{~h}$ after TACE, noncontrast helical CAT was acquired using a Light Speed 16-slice multi-detector CAT scanner (MDCT; General Electric, Milwaukee, WI). Imaging parameters included a large field of view, 5-mm slice thickness, 5-mm increment, and collimation of $20 \mathrm{~mm}$, with scans performed at 100-300 mAs and $120 \mathrm{kV}$. These unenhanced CAT scans were performed to document iodized oil distribution and exclude nontarget localization of the embolizing agent.

These 24-h noncontrast CAT scans were retrospectively reviewed for the presence or absence of renal nephrograms by one of the investigators (RWK), who has $>30$ years experience as a genitourinary radiologist and was blinded from the clinical parameters. Typically, there is no visible renal nephrogram of either the right or left kidney at $24 \mathrm{~h}$ after TACE, nor is it possible to delineate the cortex from the medulla. In these patients, the renal parenchyma on the 24-h noncontrast CAT scans was compared with each subject's most recent baseline noncontrast CAT scan (Fig. 1A). Circular regions of interest (ROIs) of the midrenal parenchyma were acquired from the baseline scan, and equivalent ROIs were acquired from 24-h post-TACE nonenhanced CAT scans. In these subjects, we compared the parenchymal values of the right kidney with those of the left kidney in each scan to determine if there was any significant difference. If there was no significant difference, the right and left parenchymal values could be combined into one data set of renal parenchymal values, termed the "expected" 24-h noncontrast CAT scans.

Two unusual distinct but different patterns of delayed nephrograms were encountered: (1) either bilateral and globally dense with or without cortical and medullary discrimination ("global" group) (Fig. 1B) or (2) with focal and wedge-shaped persistent areas of increased attenuation ("focal" group) (Fig. 2A, B). In cases of globally dense, bilateral nephrograms, in which there was visual distinction of the cortex and medulla, ROIs were acquired separately from the cortical and medullary regions. This was encountered in the majority (14 of 16 [87.5\%]) of cases. Otherwise, single ROIs of a large region of the parenchyma were obtained. For cases in which the cortex and medulla could be distinguished, we compared right with left renal attenuation values of cortex to cortex, and medulla to medulla, to determine if right and left kidneys could be combined.

The focal, wedge-shaped nephrograms were recorded as being present or absent. If present, we tabulated whether the wedge-shaped nephrogram was single or multiple and unilateral or bilateral. Because the unaffected regions of the parenchyma were visually similar, we determined whether the right and left kidneys could be combined. We
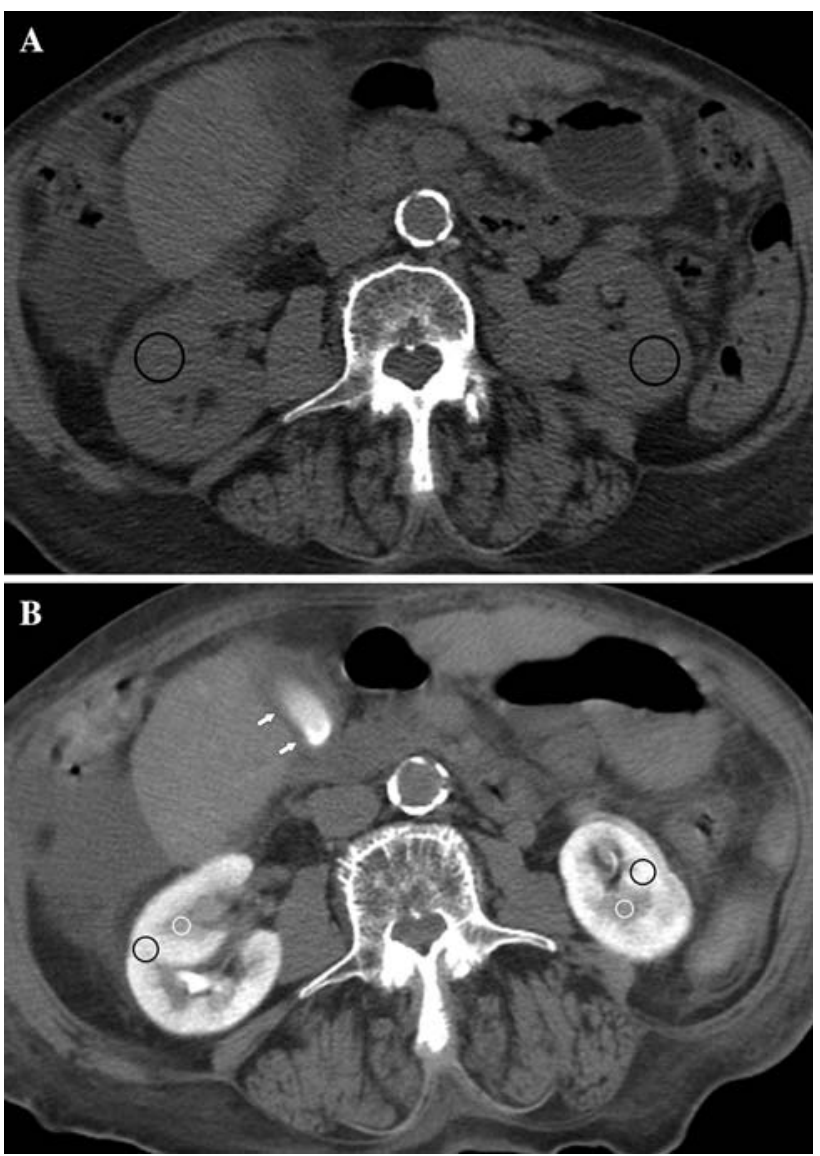

Fig. 1 Noncontrast CAT image at baseline and at $24 \mathrm{~h}$ after TACE in a 76-year-old woman with hepatocellular carcinoma and cirrhosis. A Baseline preprocedural noncontrast abdominal CAT having attenuation values of $33.8 \mathrm{HU}$ for the right renal parenchyma and $33.9 \mathrm{HU}$ for the left renal parenchyma. B Noncontrast CAT image in the same patient $24 \mathrm{~h}$ after TACE shows globally dense bilateral nephrograms. There is distinct demarcation of dense cortex and medulla as well as vicarious excretion of contrast material in the gallbladder (small white arrows). Right kidney cortical attenuation (black ROI) is $211.2 \mathrm{HU}$ and medullary attenuation (white ROI is $88.9 \mathrm{HU}$. Left kidney cortical attenuation (black ROI) is $215.5 \mathrm{HU}$ and medullary attenuation (white $\mathrm{ROI})$ is $87.9 \mathrm{HU}$

then examined whether the combined right and left "focal" data set values could be combined with the "expected" parenchymal attenuation data set. Vicarious excretion of CM on the 24-h noncontrast CAT scans was defined as the identification of fluid of increased attenuation within the gallbladder lumen (Fig. 1B).

\section{Clinical Assessment}

Retrospective review of the medical records was conducted to determine if these unusual nephrographic patterns, compared with expected nonenhancing parenchymal attenuation at 24-h after TACE, were associated with contrast volume, contrast type, fluoroscopic/procedure time, tumor size, tumor type, 

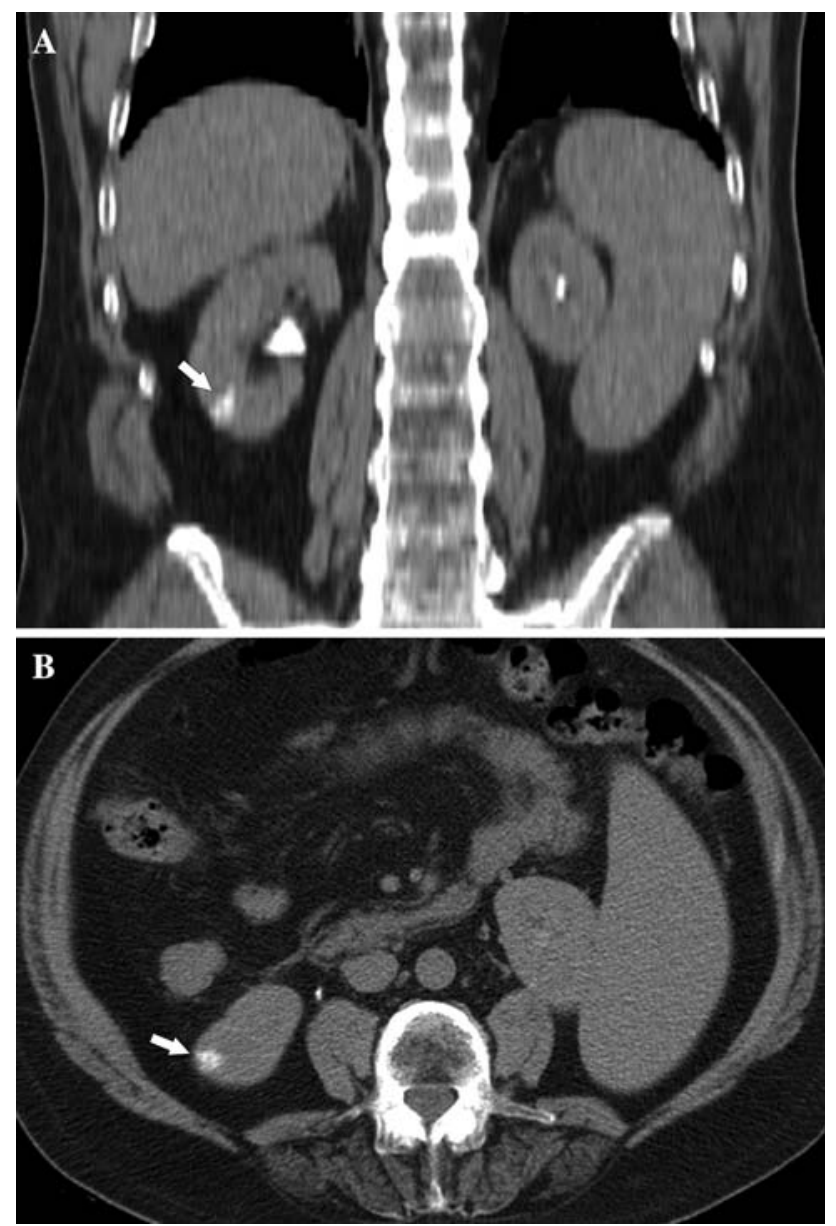

Fig. 2 Noncontrast CAT scan $24 \mathrm{~h}$ after TACE in a 54-year-old man with insulinoma metastatic to the liver. Coronal (A) and axial (B) CAT images showing a focally dense, wedge-shaped nephrogram (arrows in both figures) in the lower pole of the right kidney

age, Child-Pugh classification (which relies on biochemical and clinical criteria of bilirubin level, albumin level, prothrombin time, degree of ascites, and hepatic encephalophy), presence or absence of cirrhosis, baseline, $\mathrm{SCr}$, estimated GFR (eGFR; Modified Diet in Renal Disease estimation equation) [10], and baseline and periprocedural mean arterial blood pressures at the time of the TACE procedure (Figs. 3, 4, Table 2).

An assessment was also made of the changes in $\mathrm{SCr}$ at 24 and $48 \mathrm{~h}$ after TACE, with subsets being those patients with globally dense nephrograms, focally dense nephrograms, and the expected nonenhancing 24-h renal parenchymal attenuation (Fig. 4).

\section{Statistical Analysis}

We used the nonparametric tests of either two-sided Wilcoxon-Mann-Whitney test for nonpaired or a two-sided Wilcoxon signed-rank test for paired comparisons. To test

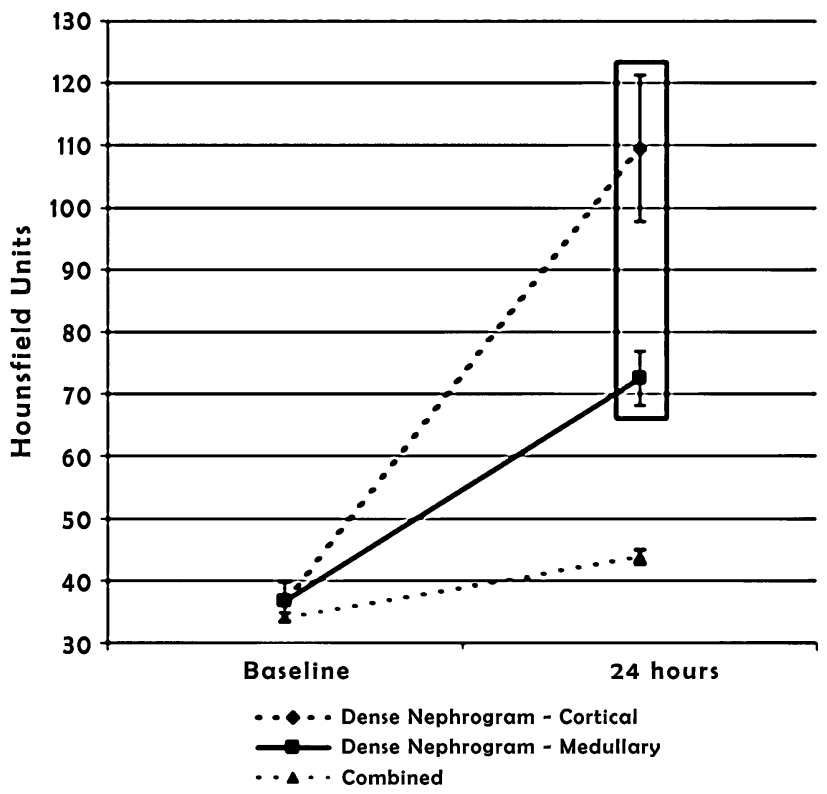

Fig. 3 Graph depicting baseline and 24-h renal attenuation (in HU) for the combined versus the globally dense nephrogram groups. There is no difference in renal parenchyma attenuation at baseline; however, there is much greater cortical and medullary attenuation $(p<0.0001$ for each) at $24 \mathrm{~h}$ compared with parenchymal attenuation in the combined group. Values shown are the means and SEMs



Fig. 4 Graph depicting changes in SCr from baseline (before TACE) to 24- and 48-h after TACE in the globally dense nephrogram versus combined nephrogram groups. The increase in SCr for the globally dense group at $24 \mathrm{~h}$ after TACE was significantly greater $(p=0.031)$ than for the combined group. There was also a near statistical difference at $48 \mathrm{~h}$ after TACE. Values shown are means and SEMs

for associations between clinical parameters and the occurrence of abnormal nephrograms on 24-h noncontrast CAT scans, we used two-sided Wilcoxon-Mann-Whitney test or two-sided Fisher's exact test; $p<0.05$ was considered statistically significant. All analyses were performed using SAS (version 9.1) software (SAS Institute, Cary, NC).

\section{Results}

Overall, abnormal nephrograms were observed in 28 of 60 (46.7\%) 24-h post-TACE nonenhanced CAT scans. Of 
these, $14(23.3 \%)$ scans were bilaterally global, and 14 (23.3\%) scans were focal. The expected parenchymal findings were noted in $32(53.3 \%)$ post-TACE CAT scans.

Focally dense nephrograms were noted unilaterally in nine, bilaterally in five, and multiple (either bilateral or unilateral) in seven scans. In contrast, attenuation values of the uninvolved parenchyma were not statistically different from the attenuation values of the baseline or 24-h noncontrast "expected" values. Therefore, for statistical analysis, the "focal" parenchymal group values were combined with the "expected" group values and termed the "combined" group values.

Persistent bilateral globally dense cortical and medullary nephrographic distinction was observed in 12 of 14 (85.7\%) scans, and globally dense but indistinguishable cortical and medullary enhancement was present in 2 of $14(14.3 \%)$ scans. Because there was no statistical difference in right to left renal cortical or medullary attenuations, these values were combined, resulting in both cortical and medullary attenuation averages derived from 24 kidneys.

On the nonenhanced, pre-TACE baseline scans, the average attenuation value of the kidneys was $34.1 \pm 4.1$ $\mathrm{HU}(\mathrm{SD})$ in the combined cases and $36.7 \pm 11.5 \mathrm{HU}$ (not significant $[\mathrm{NS}]$ ) in the cases that did have persistent global nephrograms. On the 24-h post-procedure scans, this average renal attenuation was $43.8 \pm 6.1 \mathrm{HU}$ in the combined group, whereas those with bilateral global dense nephrograms had cortical attenuation values of $109.5+43.9 \mathrm{HU}(p<0.0001)$ and medullary values of $72.5+16.3 \mathrm{HU}(p<0.0001)$ (Fig. 3). In the two circumstances of bilateral globally dense nephrograms, in which there was no distinct cortical and medullary differentiation, the cortical and medullary parenchymal attenuation values of the right kidney were 79.9 and $79.5 \mathrm{HU}$ and of the left kidney were 79.3 and $89.6 \mathrm{HU}$, respectively. These values are clearly greater than for the combined group average of $43.8 \pm 6.1 \mathrm{HU}$; however, the data set was too small to determine statistical significance.

With respect to the bilateral globally dense nephrograms, patients who had this finding after initial chemoembolization also had this finding after each subsequent procedure. Furthermore, dense nephrograms were either always present or never present in each patient. However, focal nephrograms were more sporadic in their occurrence. Two patients had focal nephrograms after the first procedure but never had them subsequently, whereas five patients had focal nephrograms only after their second or third chemoembolization. Five of the patients had focal nephrograms after their first and only chemoembolization.
Clinical Factors Associated with Persistent Globally Dense Nephrograms

When multiple clinical variables were assessed as they occurred at each procedure, presence of cirrhosis $(p=$ $0.03)$, fluoroscopic/procedure time $(p=0.028)$, lower baseline eGFR $(p<0.001)$, Child-Pugh score B or C $(p \leq 0.001)$, and lower periprocedural mean arterial blood pressure $(p=0.005)$ were significantly associated with the occurrence of bilateral globally dense nephrograms (Table 2). There was no association with tumor burden $(p=0.121$; NS), contrast medium volume $(p=0.1239$; NS), contrast medium type ( $p=0.311$; NS), or vicarious biliary excretion $(p=0.1141$; NS). Cisplatinum is a nephrotoxic chemotherapeutic agent, and a relatively high dose of $100 \mathrm{mg}$ was used in accordance with the commonly cited and used University of Pennsylvania protocol for the chemoembolization chemotherapeutic regimen, including cisplatin $(100 \mathrm{mg})$, adriamycin $(50 \mathrm{mg})$, and mitomycin $(10 \mathrm{mg})$. All patients received the same nephrotoxic drug regimen; thus, we could not correlate the use of this chemotherapeutic with the imaging and clinical findings described.

There was no statistically significant difference in contrast volume used when comparing first versus subsequent procedures when dense nephrograms were detected $(p=$ 0.5313 ) or when normal expected and focally dense nephrograms were detected ( $p=0.9332$ ). Furthermore, the type of contrast was not changed for subsequent procedures

Table 2 Variables associated with bilateral global nephrograms versus expected nephrograms seen on noncontrast CAT obtained $24 \mathrm{~h}$ after TACE

\begin{tabular}{llll}
\hline Parameters & $\begin{array}{l}\text { Combined } \\
\text { nephrogram } \\
\text { (observation) }\end{array}$ & $\begin{array}{l}\text { Bilateral global } \\
\text { nephrogram } \\
\text { (observation) }\end{array}$ & \\
\hline $\begin{array}{l}\text { Cirrhosis (yes/no) } \\
\text { Child-Pugh score }\end{array}$ & $24 / 22$ & $12 / 2$ & 0.03 \\
Noncirrhotic & 22 & 2 & $\leq 0.001$ \\
A & 1 & 1 & \\
B & 19 & 3 & 0.005 \\
C & 4 & 8 & $<0.001$ \\
MAP (mm Hg) & $90.7 \pm 13.2$ & $79.6 \pm 8.2$ & 0.12 (NS) \\
eGFR (mL/min) & $91.6 \pm 16.9$ & $71.1 \pm 19.3$ & \\
$\quad$ baseline & & $10.8 \pm 3.7$ & 0.028 \\
Tumor burden (cm) & $15.2 \pm 8.1$ & 128.0 & \\
Fluoroscopic & $37.3 \pm 22.0$ & $60.0 \pm 34.4$ & \\
$\quad$ time (min) & & & \\
CM dose (mL) & $213.5 \pm 107$ & $249.6 \pm 86$ & $p=0.12$ (NS) \\
\hline
\end{tabular}

Number of procedures $=$ unit of statistical analysis

$M A P$ mean arterial pressure

${ }^{a}$ Measured using the RECIST criteria 
based on the alteration in creatinine seen at the first procedure because there was never a large enough change in creatinine after the first procedure to warrant a change. There was also no statistically significant difference in fluoroscopy time when comparing the first procedure, in which dense nephrograms were present, with subsequent procedures $(p=0.0625)$ or when normal expected and focally dense nephrograms were detected $(p=0.9055)$.

We tested whether the focal nephrogram observation was different from the expected nephrogram relative to possible risk factors, such as catheter clotting or cholesterol emboli. This was not directly determined but indirectly suggested based on fluoroscopy time and relative qualitative assessment of atherosclerosis. A qualitative assessment of atherosclerotic disease was made from the CAT obtained before chemoembolization. The overall extent of aortic renal and mesenteric arterial atherosclerotic disease was subjectively graded as mild, moderate, or severe. Only one patient with focal nephrograms had moderate aortic and renal artery atherosclerotic disease; three patients had mild atherosclerotic disease; and the remainder of the patients had no aortic or renal atherosclerosis.

The occurrence of focal nephrographic patterns was related to fluoroscopic/procedure time $(p=0.029)$. However, focal nephrographic patterns were not associated with extent of atherosclerotic disease of the aorta or the mesenteric or renal arteries. The kidney has a tubular concentrating function that is not present in other solid viscera, such as the spleen, so it is not surprising that no other viscera demonstrated these focal densities.

\section{Relations Between CAT Nephrograms and Renal Function}

Baseline eGFR $(\mathrm{mL} / \mathrm{h})$ values were lower $(71.1+19.3$ vs. $91.6+16.9 ; p<0.001)$ in patients with persistent global nephrograms compared with those not having persistent global nephrograms. Baseline eGFR was determined on the morning of each procedure, usually near the time periprocedural hydration was initiated.

There was no significant difference in baseline, 24-h, or 48-h SCr values between the expected and focal nephrogram groups; thus, these data sets were combined. The 24-h $\mathrm{SCr}$ had a significantly higher $(p=0.031)$ increase from baseline $(1.00 \pm 0.26-1.13 \pm 0.24 \mathrm{mg} / \mathrm{L})$ in the global nephrogram group than in the combined nephrogram group $(0.87 \pm 0.32-0.88 \pm 0.3 \mathrm{mg} / \mathrm{dL})$ (Fig. 4). Baseline creatinine and GFR were always recalculated before each procedure. Our database subsets were too small to determine if baseline creatinine levels were different at each procedure. In patients with dense nephrograms, there was no difference in the degree of the change in creatinine when comparing levels after the first and subsequent procedures. However, given our small sample size, statistical significance could not be determined.

In $93 \%$ (13 of 14) of the procedures yielding global nephrograms and 41\% (19 of 46) of procedures yielding expected and focal nephrograms (combined), $\mathrm{SCr}$ was determined $48 \mathrm{~h}$ after TACE. There was a near statistical difference $(p=0.06)$ in the change of SCr from baseline between the global and combined groups at $48 \mathrm{~h}(1.0 \pm$ $0.26-1.2 \pm 0.3$ vs. $0.87 \pm 0.32-0.94 \pm 0.4$, respectively). Given that approximately one half of the expected and focal groups did not undergo a 48-h SCr because they did not require further periprocedural clinical observation for postembolization syndrome, one might also have expected a statistical difference if all subjects had received a 48-h $\mathrm{SCr}$ measurement.

In two patients seen during follow-up, creatinine levels remained slightly increased (1.1-1.4 and 0.9-1.0); in all others seen during follow-up, creatinine levels returned to baseline. One of the subjects who had globally dense nephrograms seen on CAT after TACE became oliguric, and nephrology consult was sought. This subject was vigorously hydrated and subsequently improved without the need for dialysis.

\section{Discussion}

We describe two types of delayed nephrographic patterns seen on nonenhanced CAT obtained $24 \mathrm{~h}$ after TACE. These observations were previously made only anecdotally at our institution, and we elected to assess the incidence of these findings by undertaking a systematic retrospective review of our case material. We were somewhat surprised by the relatively frequent occurrence $(46.7 \%$ overall) of these two types of nephrograms: Persistent, globally dense nephrograms occurred after 14 of 60 (23.3\%) TACE procedures and (2) wedge-like, focally persistent nephrograms, either single or multiple, occurred after 14 of 60 (23.3\%) TACE procedures. A review of the medical records was performed to determine if these unusual nephrographic patterns are associated with ARF and any specific patient clinical characteristics and/or periprocedural factors. Our intent was to investigate possible associated factors for these unusual nephrographic patterns and to assess the potential for clinical significance, which might warrant changes in periprocedural patient care.

Older et al. reported that $53 \%$ of patients who had persistent renal parenchymal enhancement on plain radiographs, which were obtained $24 \mathrm{~h}$ after angiography, had documented ARF [1]. Love et al. described noncontrast CAT scan images that demonstrated 24-h delayed cortical enhancement [3]. In their investigation, measurements averaging $>140 \mathrm{HU}$ were accompanied by clinical 
evidence of contrast-induced nephropathy (CIN). These investigators also suggested that intermediate density levels with persistent enhancement, ranging from 55 to 110 HU, represented a "subclinical decrease in renal function." Their patients had a tendency to be elderly and to have borderline chronic renal insufficiency. Jakobsen et al. described a persistent increase in the attenuation of the cortex, seen on CAT, which was attributed to pinocytosis of CM molecules into the proximal tubular cells, with the magnitude of the nonionic dimers being greater with than that of the nonionic monomers [11].

For the nephrogram of ARF, Newhouse and Pfister hypothesized that these persistent, dense nephrograms occurred due to continued glomerular filtration of CM in association with diminished cortical perfusion, which is similar to the physiology encountered in systemic hypotension or intratubular obstruction [12]. Slowed intratubular flow of the glomerular filtrate and contrast medium would be associated with increased reabsorption of sodium and water, resulting in an increased concentration of the CM molecules within the tubular lumen.

Clinical and procedural factors associated with the bilateral global nephrograms in this investigation were older patient age, presence of cirrhosis of the liver, low baseline eGFR, decreased periprocedural systemic blood pressure during or after hydration, and fluoroscopic/procedure time for the performance of TACE (Table 2). We cannot directly link CIN as the primary etiology. There was no association with contrast volume or contrast type. At times, contrast quantities may be higher than those expected from an experienced interventional radiologist. At our academic institution, some cases are performed by fellows and senior residents under the direct guidance of attending physicians with varying levels of experience (range 2-28 years in practice).

The rate of CIN between iodixanol and iohexol, as previously reported in a randomized double-blind, prospective angiographic clinical study of at-risk subjects, [13] was $>8$ times greater (3\% vs. $26 \%$ ) for the latter agent. We found no difference in ARF occurrence when comparing types of contrast. Because $>30$ different definitions of ARF have been cited in the literature [14], we used the criterion of a statistically significant change in serum creatinine (the accepted surrogate of renal function) compared with expected values in the baseline patient cohort, as described by Tepel et al. [15].

It is noteworthy that nearly all patients with globally dense nephrograms were admitted for $\geq 48 \mathrm{~h}$, whereas those without globally dense nephrograms were usually discharged within $24 \mathrm{~h}$ of the procedure. In all except one case, this prolonged admission was required for management of more severe chemoembolization syndrome, including varying degrees of nausea, emesis, discomfort, chills, and sweating. Management included continued hydration and administration of antiemetics and analgesics. One of these patients had a prolonged admission for renal insufficiency and self-limited oliguria.

Admitted patients underwent daily laboratory testing, including creatinine and liver function tests; thus, 48-h creatinine values were available. We therefore suggest that the globally dense nephrogram and renal injury may be associated with chemoembolization syndrome, which in turn is thought to be related to tumor ischemic injury and cytolysis and subsequent release of mediators. This is known to be a major reason for hospitalization after chemoembolization [16]. Lueng et al. found postembolization syndrome to be associated with gallbladder embolization and dose administered and inversely related to patients having had previous chemoembolization. We hypothesize that postembolization syndrome may also be related to renal injury.

Significant changes in SCr occurred between baseline and $24 \mathrm{~h}$ after TACE in subjects who had bilateral globally dense nephrograms. There was a near statistically significant change at $48 \mathrm{~h}$ between groups as well. Only approximately one half of the combined group received 48 SCrs because they were more often likely to be clinically stable enough for discharge at $24 \mathrm{~h}$. The baseline eGFR was lower in the global nephrogram group than the combined group, suggesting higher vulnerability to ARF.

We believe that the finding of delayed, globally dense nephrograms is possibly a more sensitive indicator of renal compromise than changes in SCr. Limitations of $\mathrm{SCr}$ as an indicator of renal injury are well known [10]. In patients with normal renal function, there must be at least a $50 \%$ decrease in GFR before the SCr changes significantly. Furthermore, there is a delay in the occurrence of $\mathrm{SCr}$ increases related to the timing of ARF. Shemesh et al. demonstrated that endogenous creatinine is hypersecreted progressively by remnant renal tubules in patients with glomerular disease [17]. These investigators suggested that use of creatinine as a marker of renal function in glomerulopathic patients will result in "gross and unpredictable overestimates of the GFR."

One of our patients developed oliguric renal failure that required more aggressive clinical care and the involvement of the Nephrology Service. This patient was vigorously hydrated and subsequently improved without requiring dialysis. However, recent reports in the nephrologic literature suggest that even modest transient changes in SCr may signal an increased risk of adverse outcomes. For example, a consecutive sampling of 19,982 adults admitted to an urban academic medical center showed that modest changes in SCr were significantly associated with increased mortality, length of hospital stay, and cost, even after adjustment for age, sex, severity of illness, and chronic kidney 
disease [18] Ritz and McClellan noted that minor renal dysfunction, as reflected by an increase in $\mathrm{SCr}$ (or, more precisely, in eGFR) and/or albuminuria or trace proteinuria, has a major impact on cardiovascular risk [19]. Our current investigation is too limited to assess long-term outcome, and the overwhelming medical condition, i.e., terminal neoplastic disease, would be a major confounding factor in determining this possibility in our patient population.

If he or she sees these unique nephrographic findings on postchemoembolization CAT, the radiologist plays an important role in the diagnosis of possible renal injury. The finding of a persistent nephrogram may precede the development of oliguria or a detectable increase in $\mathrm{SCr}$, which might warrant a significant change in clinical care. Aggressive hydration to establish a urinary output of at least 3 litres/24 h and close surveillance of clinical chemistries have been recommended at the earliest suggestion of renal insufficiency after tumoricidal therapies with suspected tumor lysis syndrome [20]. Administration of allopurinol before cytotoxic therapy has been recommended for prevention of ARF associated with tumor lysis [21]. In our study, however, the globally dense nephrograms were not associated with higher tumor burden. We cannot substantiate tumor lysis as a causative factor of ARF in our study.

We observed a statistically significant association between the dense nephrograms and the presence of cirrhosis and the severity of hepatic disease as indicated by Child-Pugh classification. This may be related to exacerbated hepatorenal syndrome. Hepatorenal syndrome is defined as renal failure in patients with severely compromised liver function in the absence of clinical, laboratory, or anatomic evidence of other causes of renal failure [21]. It resembles prerenal failure but does not respond to volume replacement. No specific treatment exists; management is conservative; and prognosis is poor. Epstein et al. found renal xenon washout patterns, suggesting that decreased renal perfusion in cirrhotic patients with hepatorenal syndrome is secondary to active vasoconstriction and cortical ischemia [22]. Possible mediators of vasoconstriction include serotonin (5-hydroxytryptamine) and angiotensin. It is possible that treatment with vasodilators may reverse hepatorenal-associated failure and may have a role in these patients when dense nephrograms are present on CAT after chemoembolization.

Segmental, wedge-shaped nephrograms on delayed imaging studies have only rarely been reported. Anecdotal case reports were described by Ishikawa et al. in 1985 [4, 5], by Pazmino et al. in 1983 [6], by Braedel et al. in 1987 [23], and by Trueba-Arguinarena et al. in 1997 [24].

We found the occurrence of wedge-shaped nephrograms in 14 of $60(23.3 \%)$ noncontrast CAT scans obtained $24 \mathrm{~h}$ after TACE. We did not find an associated statistically significant change in SCr. We postulate that these wedge- shaped nephrograms represent trapped CM in the tubular lumen secondary to segmental perfusion deficits, i.e., either embolic events or segmental vasoconstriction. One candidate for the latter hypothesis is the release of endogenous vasoconstrictors, such as angiotensin II. When CM is administered during the TACE procedure, there is-as usual-the initial development of bilateral global nephrograms. If embolic events occur during the catheterization procedure, or if vasoactive endogenous hormones are liberated, then segmental areas of the renal parenchyma may contain trapped CM. The well-perfused parenchyma clears the CM, which has a biologic half-time of approximately $1.5 \mathrm{~h}$. However, in the areas with segmental ischemia, the $\mathrm{CM}$ does not empty, and sodium and water continue to be reabsorbed from the tubular lumen. This results in accentuation of the focally ischemic regions compared with the normal parenchyma, which has cleared the CM. Cholesterol emboli have indeed been noted to occur during angiographic procedures [25, 26]. Furthermore, clots can form around a catheter during angiographic procedures. Researchers have shown that angiographic techniques can lead to the release of particles, presumably made up of bits of clot and fatty plaque material, into the blood [26]. It has also been reported that cholesterol emboli are a known cause of ARF [25].

Our study is limited by our relatively small number of patients and by numerous underlying clinical factors that confounded the data. Review of the intraprocedural images did not suggest iatrogenic or technical causes, such as nontarget renal artery embolization during chemoembolization or cholesterol/atherosclerotic embolization, although it may be impossible to entirely exclude this as possibly occurring during the procedure or with retrospective review of the angiographic images. We did not demonstrate an association with the degree of aortic, mesenteric, or renal artery atherosclerotic changes with either the focal or diffuse dense nephrograms. Continued data collection from a larger database of patients can help isolate these factors. Assessing imaging findings in patients who have undergone different types of angiographic procedures, such as coronary angiography, may help clarify the role of underlying hepatic disease and renal insufficiency versus other factors, such as atherosclerotic vasculopathy, leading to ischemic renal injury. Furthermore, animal models can be used to elucidate the effect of renal emboli on subsequent nephrographic patterns.

In conclusion, we describe two distinct patterns of persistent delayed nephrograms in patients receiving noncontrast CAT scans $24 \mathrm{~h}$ after TACE. We suggest that these observations could be sensitive indicators of alterations in renal physiology and the occurrence of ARF. Furthermore, the detection of these findings might warrant changes in periprocedural and subsequent clinical care. One might 
consider making changes during subsequent chemoembolization, such as decreased use of nephrotoxic chemotherapeutics and use of the described sodium bicarbonate regimen, with subsequent close monitoring of urine output. A low threshold for the consultation of the Nephrology Service might be advocated. There has been interest in the use of intrarenal fenoldopam, using the Benephit System (Angiodynamics, Freemont, CA), for renal infusion therapy after angiographic procedures in patients at risk for developing CIN [27]. Although this phenomenon is not thought to represent CIN, patients with these findings who do develop a clinically significant decrease in renal function might benefit from this therapy. Recently, there has been increasing use of doxorubicin drug-eluting beads (LC Bead; Angiodynamics, Freemont, CA). Because no ethiodol is used in this regimen, follow-up nonenhanced CAT is not commonly performed on the day after chemoembolization; thus, these findings would not be detected. Furthermore, it is not known what effect altered pharmacokinetics and decreased systemic chemotherapeutic will have on these findings. Interventional and diagnostic radiologists should be aware of these unique nephrographic findings on CAT after TACE and their possible clinical implications.

Open Access This article is distributed under the terms of the Creative Commons Attribution Noncommercial License which permits any noncommercial use, distribution, and reproduction in any medium, provided the original author(s) and source are credited.

\section{References}

1. Older RA, Miller JP, Jackson DC et al (1976) Angiographically induced renal failure and its radiographic detection. AJR Am J Roentgenol 126:1039-1045

2. Older RA, Korobkin M, Cleeve DM et al (1980) Contrastinduced acute renal failure: persistent nephrogram as clue to early detection. AJR Am J Roentgenol 134:339-342

3. Love L, Lind JA, Olson MC (1989) Persistent CT nephrogram with significance in the diagnosis of contrast nephropathy. Radiology 172:125-129

4. Ishikawa I, Saito Y, Shinoda A et al (1981) Evidence for patchy renal vasoconstriction in man: observation by CT scan. Nephron 27:31-34

5. Ishikawa I, Tateishi K, Onouchi Z et al (1985) Persistent wedgeshaped contrast enhancement of the kidney. Urol Radiol 7:45-47

6. Pazmiño P, Pyatt R, Williams E et al (1983) Computed tomography in renal ischemia. J Comput Assist Tomogr 7:102-105

7. Therasse P, Arbuck SG, Eisenhauer EA et al (2000) New guidelines to evaluate the response to treatment in solid tumors. European Organization for Research and Treatment of Cancer, National Cancer Institute of the United States, National Cancer Institute of Canada. J Natl Cancer Inst 92:205-216

8. Solomon B, Soulen MC, Baum RA et al (1999) Chemoembolization of hepatocellular carcinoma with cisplatin, doxorubicin,
mitomycin-C, ethiodol, and polyvinyl alcohol: prospective evaluation of response and survival in a U.S. population. J Vasc Interv Radiol 10:793-798

9. Merten GJ, Burgess WP, Gray LV et al (2004) Prevention of contrast-induced nephropathy with sodium bicarbonate: a randomized controlled trial. JAMA 291(19):2328-2334

10. Levey A, Bosch JP, Lewis JB et al (1999) A more accurate method to estimate glomerular filtration rate from serum creatinine: a new prediction equation. Ann Intern Med 130:461-470

11. Jakobsen JA, Lundby B, Kristoffersen DT et al (1992) Evaluation of renal function with delayed CT after injection of nonionic monomeric and dimeric contrast media in healthy volunteers. Radiology 182:419-424

12. Newhouse JA, Pfister RC (1979) The nephrogram. Radiol Clin North Am 17:213-225

13. Aspelin P, Aubry P, Fransson S-G et al (2003) Nephrotoxic effects in high-risk patients undergoing angiography. $\mathrm{N}$ Engl J Med 348:491-499

14. Bellomo R, Kellum JA, Ronco C (2007) Defining and classifying acute renal failure: from advocacy to concensus and validation of the RIFLE Criteria. Intens Care Med 33:409-413

15. Tepel M, van der Giet M, Schwartzfeld C et al (2000) Prevention of radiographic-contrast-agent-induced reductions in renal function by acetycysteine. N Engl J Med 343:180-184

16. Leung DA, Goin JE, Sickles C et al (2001) Determinants of postembolization syndrome after hepatic chemoembolization. J Vasc Interv Radiol 12:321-326

17. Shemesh O, Golbetz H, Kriss JP et al (1985) Limitations of creatinine as a filtration marker in glomerulopathic patients. Kidney Int 28:830-838

18. Chertow GM, Burdick E, Honour M et al (2005) Acute kidney injury, mortality, length of stay, and costs in hospitalized patients. J Am Soc Nephrol 16:3365-3370

19. Ritz E, McClellan WM (2004) Overview: increased cardiovascular risk in patients with minor renal dysfunction: an emerging issue with far-reaching consequences. J Am Soc Nephrol 15:513-516

20. Sakamoto N, Monzawa S, Nagano H et al (2007) Acute tumor lysis syndrome caused by transcatheter oily chemoembolization in a patient with a large hepatocellular carcinoma. Cardiovasc Intervent Radiol 30:508-511

21. Portilla D, Shaver MJ (2004) Acute renal failure. In: Andreoli TE (ed) Cecil essentials of medicine, 6th edn. Saunders, Philadelphia, pp 291-299

22. Epstein NM, Berk DP, Hollenberg NK et al (1970) Renal failure in the patient with cirrhosis. The role of active vasoconstriction. Am J Med 49:175-185

23. Braedel HU, Schindler E, Cullen P et al (1987) Demonstration of residual contrast medium in renal parenchyma using computerized tomography. J Urol 137:11-14

24. Trueba Arguiñarena FJ, Fuertes Alija JJ, Perez Homs M et al (1997) Unilateral tubular obstruction: an unusual complication of urographic contrast media. AJR Am J Roentgenol 169:1585-1587

25. Vidt DG (1997) Cholesterol emboli, a common cause of renal failure. Annu Rev Med 48:375-385

26. Keeley EC, Grines CL (1998) Scraping of aortic debris by coronary guiding catheters. A prospective evaluation in 1,000 cases. J Am Coll Cardiol 32:1861-1865

27. Ng MK, Tremmel J, Fitzgerald PJ et al (2006) Selective renal arterial infusion of fenoldopam for the prevention of contrastinduced nephropathy. J Interv Cardiol 19:75-79 